\title{
Preparation and Characterization of Superparamagnetic Molecularly Imprinted Polymers for Selective Adsorption and Separation of Vanillin in Food Samples
}

\author{
Fangjian Ning, ${ }^{\dagger}$ Hailong Peng, ${ }^{*}, \dagger$, Liling Dong, $^{\dagger}$ Zhong Zhang, ${ }^{\S}$ Jinhua $\mathrm{Li}^{\S}{ }^{\S}$ Lingxin Chen, ${ }^{\S}$ \\ and Hua Xiong*, $\dagger$
}

\author{
${ }^{\dagger}$ State Key Laboratory of Food Science and Technology, Nanchang University, Nanchang, Jiangxi 330047, People’s Republic of China \\ ${ }^{\ddagger}$ Department of Chemical Engineering, Nanchang University, Nanchang, Jiangxi 330031, People’s Republic of China \\ ${ }^{\S}$ Key Laboratory of Coastal Environmental Processes and Ecological Remediation, Yantai Institute of Coastal Zone Research, Chinese \\ Academy of Sciences, Yantai, Shandong 264003, People's Republic of China
}

\begin{abstract}
Novel water-compatible superparamagnetic molecularly imprinted polymers (M-MIPs) were prepared by coating superparamagnetic $\mathrm{Fe}_{3} \mathrm{O}_{4}$ nanoparticles with MIPs in a methanol-water reaction system. The M-MIPs were used for the selective adsorption and separation of vanillin from aqueous solution. The M-MIPs were characterized by X-ray powder diffraction (XRD), Fourier transform infrared (FTIR) spectroscopy, thermogravimetric analysis (TGA), vibrating sample magnetometry (VSM), and scanning electron microscopy (SEM). Results indicated that a core-shell structure of M-MIPs was obtained by coating a layer of silica and MIPs on the surface of the $\mathrm{Fe}_{3} \mathrm{O}_{4}$ nanoparticles. The obtained M-MIPs possess a loose and porous structure and can be rapidly separated from the solution using a magnet. The adsorption experiments showed that the binding capacity of the M-MIPs was significantly higher than that of the superparamagnetic non-molecularly imprinted polymers (M-NIPs). Meanwhile, the adsorption of M-MIPs reached equilibrium within 100 min, and the apparent maximum adsorption quantity $\left(Q_{\max }\right)$ and dissociation constant $\left(K_{\mathrm{d}}\right)$ were $64.12 \mu \mathrm{mol} \mathrm{g}-1$ and $58.82 \mu \mathrm{mol} \mathrm{L} \mathrm{L}^{-1}$, respectively. The Scatchard analysis showed that homogeneous binding sites were formed on the M-MIP surface. The recoveries of 83.39-95.58\% were achieved when M-MIPs were used for the pre-concentration and selective separation of vanillin in spiked food samples. These results provided the possibility for the separation and enrichment of vanillin from complicated food matrices by M-MIPs.
\end{abstract}

KEYWORDS: superparamagnetism, water-compatible, magnetic molecularly imprinted polymers, vanillin, selective adsorption

\section{INTRODUCTION}

Vanillin (3-methoxy-4-hydroxybenzaldehyde) is an important flavor enhancer that is used to improve the fragrance of commercial foods, cosmetics, and pharmaceutical products. ${ }^{1-3}$ Synthetic vanillin, instead of natural vanillin, covers most of the market requirement because of its simple synthesis process with low-cost starting materials. Vanillin is used as an additive, particularly in infant formula foods, to conceal the unpleasant original flavor to increase the appetite of babies. However, excessive intake of vanillin may lead to headache, ill feeling, vomiting, expiratory dyspnea, or even liver and kidney damage. ${ }^{4}$ Therefore, determining the appropriate dosage and controlling the intake of vanillin are important.

Several methods for detecting vanillin have been reported, namely, ultraviolet-visible (UV-vis) spectrometry (UV), ${ }^{5,6}$ high-performance liquid chromatography (HPLC), ${ }^{7,8}$ liquid chromatography-mass spectrometry (LC-MS), ${ }^{9,10}$ and gas chromatography-mass spectrometry (GC-MS). ${ }^{11}$ UV-based methods do not involve complex pretreatment processes, easily detect vanillin, but are insensitive to crude samples. Although chromatographic methods exhibit high sensitivity and specificity, they consume a significant amount of time in the pretreatment of samples and demand highly sophisticated instrumentation. ${ }^{6,12}$ Therefore, the development of a sensitive and specific method for convenient pretreatment, separation, and enrichment of vanillin from commercial samples is necessary.

Molecular imprinting technology is an attractive method for molecular recognition with high sensibility and selectivity. This technology has been extensively applied in various areas, including biomimetic sensors, ${ }^{13}$ solid-phase extraction, ${ }^{14}$ chemical and biochemical separation, ${ }^{15}$ and mimic enzyme catalysis. ${ }^{16}$ Three-dimensional cavities formed during the synthesis process of molecularly imprinted polymers (MIPs) are complementary to the template in terms of shape, size, and functional groups, thereby enabling the re-identification of target molecules. In our previous study, ${ }^{17}$ MIPs for vanillin were prepared by precipitation polymerization and applied to solid-phase extraction. However, polymers have some disadvantages, such as weak binding capacity, slow binding kinetics, and complex separation processes. Moreover, Wang et al. $^{18}$ used bulk polymerization to synthesize vanillinimprinted polymers and investigated the influence of different porogen agents. However, in this method, a series of complicated post-treatments, including crushing, grinding, sieving, and centrifugation, are necessary. Given the heteroge-

Received: August 29, 2014

Revised: October 28, 2014

Accepted: October 28, 2014

Published: October 28, 2014 
neous internal distribution of binding sites, the mass transfer of target molecules from solution to cavities is obstructed by the adsorption of the template on the surface of MIPs, which causes low binding affinity.

To overcome these problems, a surface-imprinting technique combined with magnetic nanoparticles [superparamagnetic MIPs (M-MIPs)] has been applied recently, ${ }^{19-21}$ in which most of the binding sites on the surface of polymers are exposed to facilitate the recognition and removal of the template. In addition, magnetic support can be easily isolated from the real samples using a magnet. However, traditional polymerization processes of MIPs are conducted in organic phase, thereby restricting its application in aqueous systems. In recent years, water-compatible MIPs have been developed. For example, tramadol-imprinted polymers embedding $\mathrm{SiO}_{2}-$ $\mathrm{Fe}_{3} \mathrm{O}_{4}$ nanoparticles were prepared in double-distilled water and then used to separate tramadol from human urine samples. The obtained MMIPNPs exhibit high sensitivity and low detection limits. $^{22}$ Tao Jing et al. ${ }^{23}$ prepared lysozymeimprinted magnetic nanoparticles immersed in phosphate buffer $\left(0.2 \mathrm{~mol} \mathrm{~L} \mathrm{~L}^{-1}, \mathrm{pH} 6.2\right)$ and reused them 5 times. The high adsorption capability and high saturation magnetization of the lysozyme-imprinted magnetic nanoparticles enable their efficient separation from the crude sample. However, to the best of our knowledge, only a few studies on M-MIPs of vanillin have been conducted.

In this paper, we report the use of a simple method to prepare core-shell M-MIPs in a methanol-water reaction system. The obtained M-MIPs were used for the separation and pre-concentration of vanillin. The $\mathrm{Fe}_{3} \mathrm{O}_{4}$ nanoparticles were synthesized by the alkaline co-precipitation method and then coated by a $\mathrm{SiO}_{2}$ layer, in which the surface was modified by 3methacryloxypropyltrimethoxysilane (MPS) to form end vinyl bonds that provided reaction sites to induce the occurrence of imprinted polymerization on the surface of the $\mathrm{Fe}_{3} \mathrm{O}_{4} @ \mathrm{SiO}_{2} @$ MPS. Finally, M-MIP particles were prepared for the separation and enrichment of vanillin from the aqueous solution before further HPLC-UV analysis. These M-MIPs are watercompatible and highly selective, and they exhibit fast binding kinetics and can be rapidly separated.

\section{EXPERIMENTAL SECTION}

Reagents and Materials. Vanillin, methyl vanillin, ethyl vanillin, ferrous sulfate heptahydrate $\left(\mathrm{FeSO}_{4} \cdot 7 \mathrm{H}_{2} \mathrm{O}\right)$, anhydrous ferric chloride $\left(\mathrm{FeCl}_{3}\right)$, ammonia $\left(\mathrm{NH}_{3} \cdot \mathrm{H}_{2} \mathrm{O}\right)$, ethanol, methanol, and acetic acid were all purchased from Sinopharm Chemical Reagent Co., Ltd. (Shanghai, China). Methacrylic acid (MAA), ethylene glycol dimethacrylamide (EGDMA), 2,2-azobis(isobutyronitrile) (AIBN), and MPS were obtained from Sigma (Shanghai, China). Tetraethoxysilane (TEOS) was obtained from Aladdin (Shanghai, China). All of these chemicals were of analytical reagent grade.

Preparation of $\mathrm{Fe}_{3} \mathrm{O}_{4} @ \mathrm{SiO}_{2}$ Nanoparticles. The $\mathrm{Fe}_{3} \mathrm{O}_{4}$ magnetic nanoparticles were synthesized via the alkaline coprecipitation method. Briefly, $1.63 \mathrm{~g}$ of $\mathrm{FeCl}_{3}$ and $1.45 \mathrm{~g}$ of $\mathrm{FeSO}_{4}$. $7 \mathrm{H}_{2} \mathrm{O}$ were dissolved in $100 \mathrm{~mL}$ of distilled water with vigorous stirring for $20 \mathrm{~min}$ under a nitrogen atmosphere. When the temperature was increased to $60{ }^{\circ} \mathrm{C}, 5 \mathrm{~mL}$ of $\mathrm{NH}_{3} \cdot \mathrm{H}_{2} \mathrm{O}(25-28 \%$, $\mathrm{w} / \mathrm{w})$ was injected into the mixture rapidly, and then the solution immediately turned black. The reaction was allowed to proceed for 60 min under a nitrogen atmosphere. The black product was separated from the liquid using a permanent magnet and then washed with distilled water several times until the $\mathrm{pH}$ of the washing water was neutral.

The obtained $\mathrm{Fe}_{3} \mathrm{O}_{4}$ nanoparticles were dispersed homogeneously into $100 \mathrm{~mL}$ of distilled water by sonication for $20 \mathrm{~min}$. The magnetic dispersion $(10 \mathrm{~mL})$ was mixed with $80 \mathrm{~mL}$ of ethanol, followed by the addition of $5 \mathrm{~mL}$ of ammonia $(25-28 \%$, w/w). Subsequently, $1 \mathrm{~mL}$ of TEOS was added drop by drop, and the reaction was maintained for 6 $\mathrm{h}$ at $40{ }^{\circ} \mathrm{C}$. The obtained $\mathrm{Fe}_{3} \mathrm{O}_{4} @ \mathrm{SiO}_{2}$ particles were collected using a magnet, rinsed alternately with ethanol and water 3 times, and then dried under vacuum for $24 \mathrm{~h}$.

MPS, a silane coupling agent, was used to modify the silica surface to enhance the binding capacity between the magnetic particles and the MIPs. In this procedure, $500 \mathrm{mg}$ of $\mathrm{Fe}_{3} \mathrm{O}_{4} @ \mathrm{SiO}_{2}$ particles was dispersed in $50 \mathrm{~mL}$ of anhydrous toluene by sonication for $20 \mathrm{~min}$, followed by the addition of $10 \mathrm{~mL}$ of MPS. The reaction was conducted with reflux for $24 \mathrm{~h}$ at $50{ }^{\circ} \mathrm{C}$ under a nitrogen atmosphere. The resulting $\mathrm{Fe}_{3} \mathrm{O}_{4} @ \mathrm{SiO}_{2}-\mathrm{MPS}$ was thoroughly washed with toluene and dried under vacuum.

Preparation of M-MIPs. The M-MIPs were synthesized by dissolving $0.68 \mathrm{mmol}$ of vanillin (as template) and $2.72 \mathrm{mmol}$ of MAA (as a functional monomer) in $30 \mathrm{~mL}$ of methanol/water $(9: 1, \mathrm{v} / \mathrm{v}$ ) solution. After vortex oscillation, the mixture was stored under refrigeration at $4{ }^{\circ} \mathrm{C}$ overnight to form hydrogen bonds between vanillin and MAA. Subsequently, $100 \mathrm{mg}$ of modified $\mathrm{Fe}_{3} \mathrm{O}_{4} @ \mathrm{SiO}_{2}, 6.8$ mmol of EGDMA (as a cross-linker), and $30 \mathrm{mg}$ of AIBN (as an initiator) were dispersed in $20 \mathrm{~mL}$ of methanol/water $(9: 1, \mathrm{v} / \mathrm{v})$ by sonication for $25 \mathrm{~min}$, followed by the addition of the vanillin-MAA mixture. After nitrogen gas injection, the solution was mixed at $50{ }^{\circ} \mathrm{C}$ for $8 \mathrm{~h}$, then kept at $60{ }^{\circ} \mathrm{C}$ for $24 \mathrm{~h}$, and further aged for $6 \mathrm{~h}$ at $80^{\circ} \mathrm{C}$. The polymer products were eluted with methanol/acetic acid (9:1, v/ $\mathrm{v}$ ) in a Soxhlet extractor for $48 \mathrm{~h}$ to remove the templates. Finally, the obtained M-MIPs were dried under vacuum for $24 \mathrm{~h}$. Superparamagnetic non-MIPs (M-NIPs, as a control) were prepared using the method described previously without the addition of vanillin.

Characterization. Fourier transform infrared (FTIR) spectra were recorded using the FTIR spectrophotometer (Nicolet 5700, Thermo Electron Corporation, Waltham, MA). X-ray powder diffraction (XRD) patterns were recorded using an XRD analyzer (D8-FOCUS, Bruker, Karlsruhe, Germany). Transmission electron microscopy (TEM) images were obtained on a JEOL (JEM-2010HR, Japan) transmission electron microscope. The magnetic properties were examined by vibrating sample magnetometry (VSM, 7407, Lakeshore, Westerville, $\mathrm{OH}$ ). The amounts of analytes were determined by a HPLC-UV (Agilent Technolologies 1260 Infinity) method. The ZORBAX SB-C18 column with a $250 \times 4.6 \mathrm{~mm}$ inner diameter was used as the analytical column. HPLC-UV conditions employed were as follows: mobile phase, methanol/water/acetic acid (3:7:0.05, v/v/ v); flow rate, $0.3 \mathrm{~mL} \mathrm{~min}^{-1}$; and detection wavelength, $279 \mathrm{~nm}$, under room temperature.

Binding Experiment. To evaluate the adsorption capacity of MMIPs or M-NIPs, $10 \mathrm{mg}$ of particles was dispersed in $5 \mathrm{~mL}$ of vanillin solution with concentrations from 0.04 to $0.15 \mathrm{mmol} \mathrm{L}^{-1}$. The mixture was incubated at room temperature for $24 \mathrm{~h}$ and then separated by a magnet to determine the concentration of vanillin in supernatant solution.

The M-MIPs or M-NIPs $(20 \mathrm{mg})$ were added to $30 \mathrm{~mL}$ of 0.08 mmol L $\mathrm{L}^{-1}$ vanillin solution to investigate their kinetic adsorption properties. The mixture was incubated in a shaker at room temperature, and samples were obtained at different times. The microspheres were then separated by a magnet to determine the residual concentration of vanillin using a UV spectrophotometer at $279 \mathrm{~nm}$.

Selectivity experiments were conducted using vanillin, methyl vanillin, and ethyl vanillin as structural analogues. M-MIPs $(20 \mathrm{mg})$ or M-NIPs $(20 \mathrm{mg})$ were dispersed into $2.0 \mathrm{~mL}$ methanol-water solutions containing $0.08 \mathrm{mmol} \mathrm{L}^{-1}$ vanillin, methyl vanillin, and ethyl vanillin. The amount of vanillin, methyl vanillin, and ethyl vanillin in the supernatant were determined by UV after shaking for $12 \mathrm{~h}$ at room temperature. The recognition ability of M-MIPs was evaluated using the imprinting factor $(\alpha)$, which is defined in the following equation:

$$
\alpha=Q_{\mathrm{MIP}} / Q_{\mathrm{NIP}}
$$

where $Q_{\text {MIP }}$ and $Q_{\text {NIP }}$ are the adsorption amounts of the templates or analogues on M-MIPs and M-NIPs, respectively. 

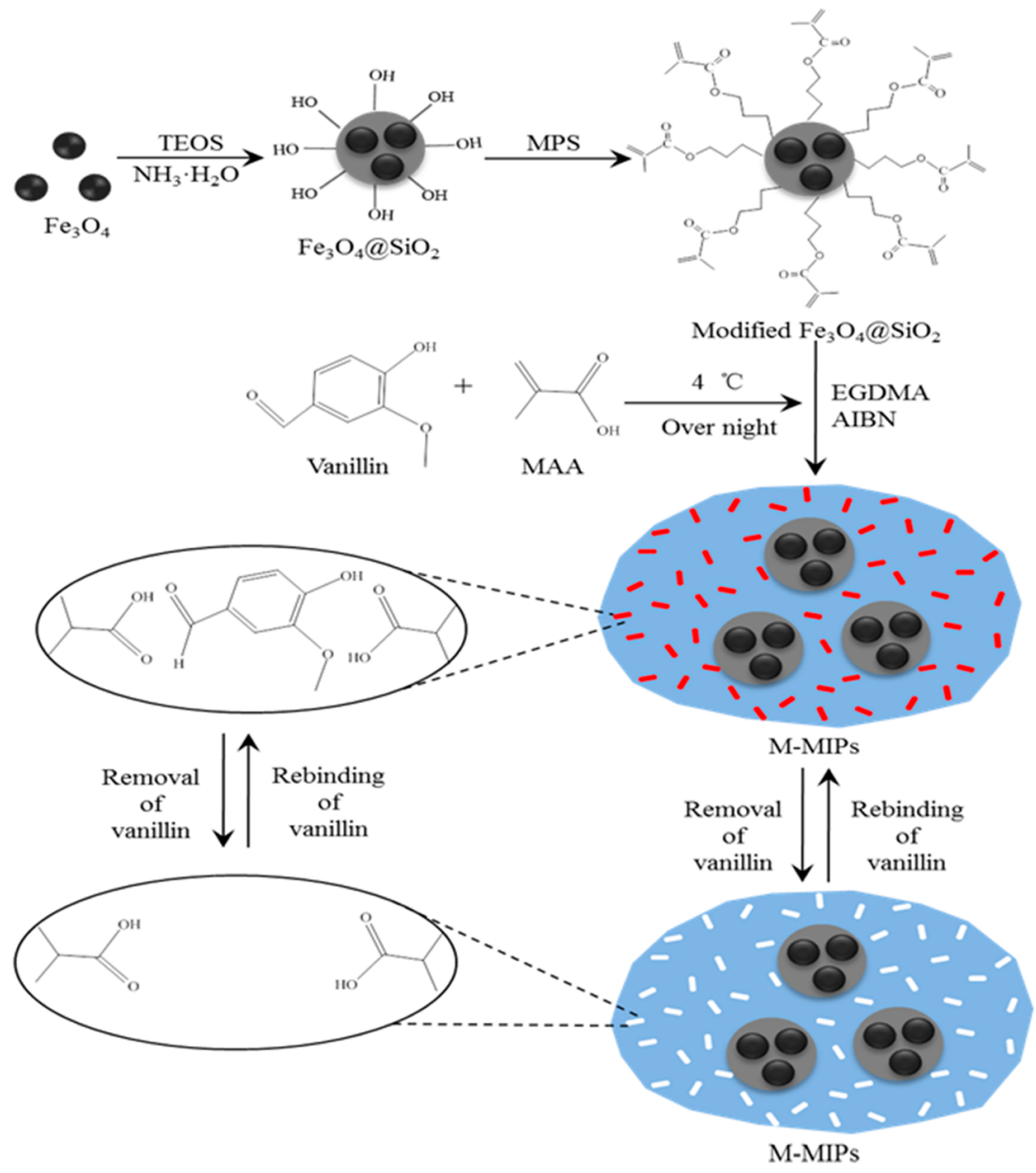

Figure 1. Synthesis schematic of M-MIPs.
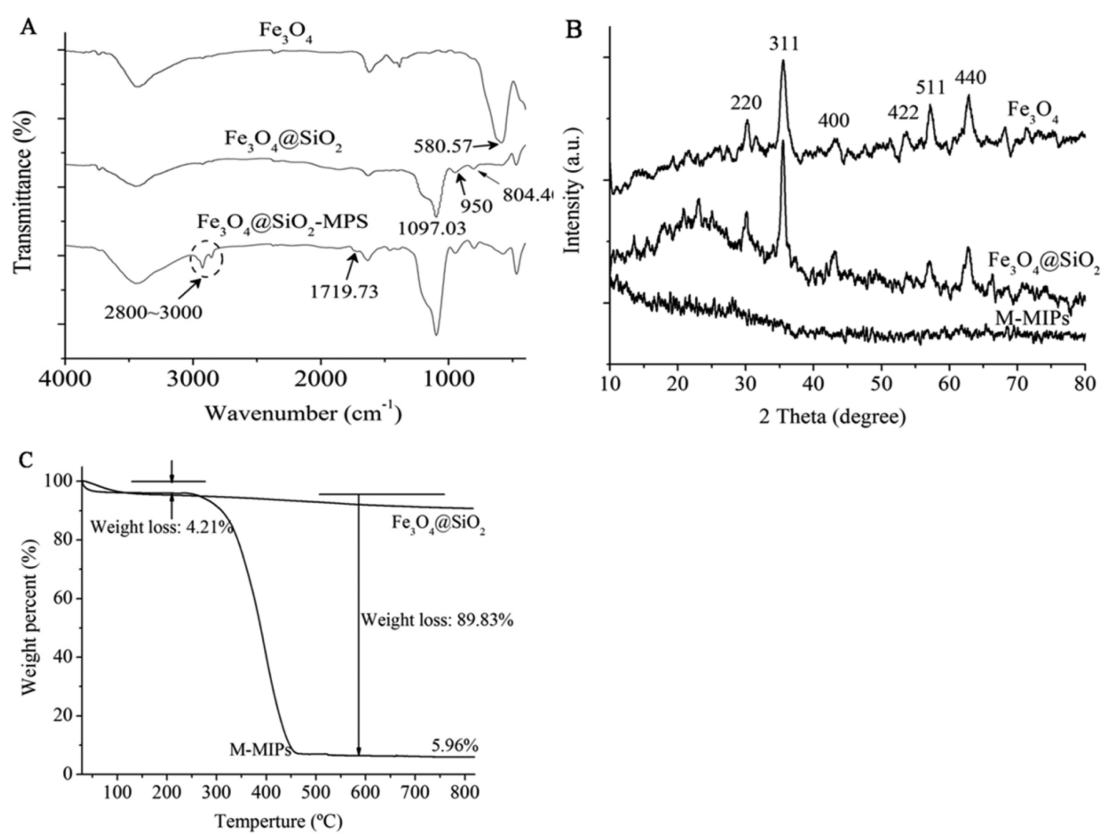

Figure 2. (A) FTIR spectra of $\mathrm{Fe}_{3} \mathrm{O}_{4} @ \mathrm{SiO}_{2}, \mathrm{MPS}$, and $\mathrm{Fe}_{3} \mathrm{O}_{4} @ \mathrm{SiO}_{2}-\mathrm{MPS}$, (B) XRD of $\mathrm{Fe}_{3} \mathrm{O}_{4}, \mathrm{Fe}_{3} \mathrm{O}_{4} @ \mathrm{SiO}_{2}$, and M-MIPs, and (C) thermogravimetric curves of $\mathrm{Fe}_{3} \mathrm{O}_{4} @ \mathrm{SiO}_{2}$ and M-MIPs.

Analysis of Vanillin in Real Samples. Several commercial food samples (bread, milk powder, biscuit, and chocolate) were purchased from the local market in Nanchang. The food samples were ground to a fine powder using a mortar and pestle. Food samples $(2 \mathrm{~g})$ unspiked or spiked with different concentrations of vanillin standard solutions $\left(2 \mathrm{~mL}\right.$ or $0.01,0.04$, or $\left.0.08 \mathrm{mmol} \mathrm{L}^{-1}\right)$ and using water $(20 \mathrm{~mL})$ were 

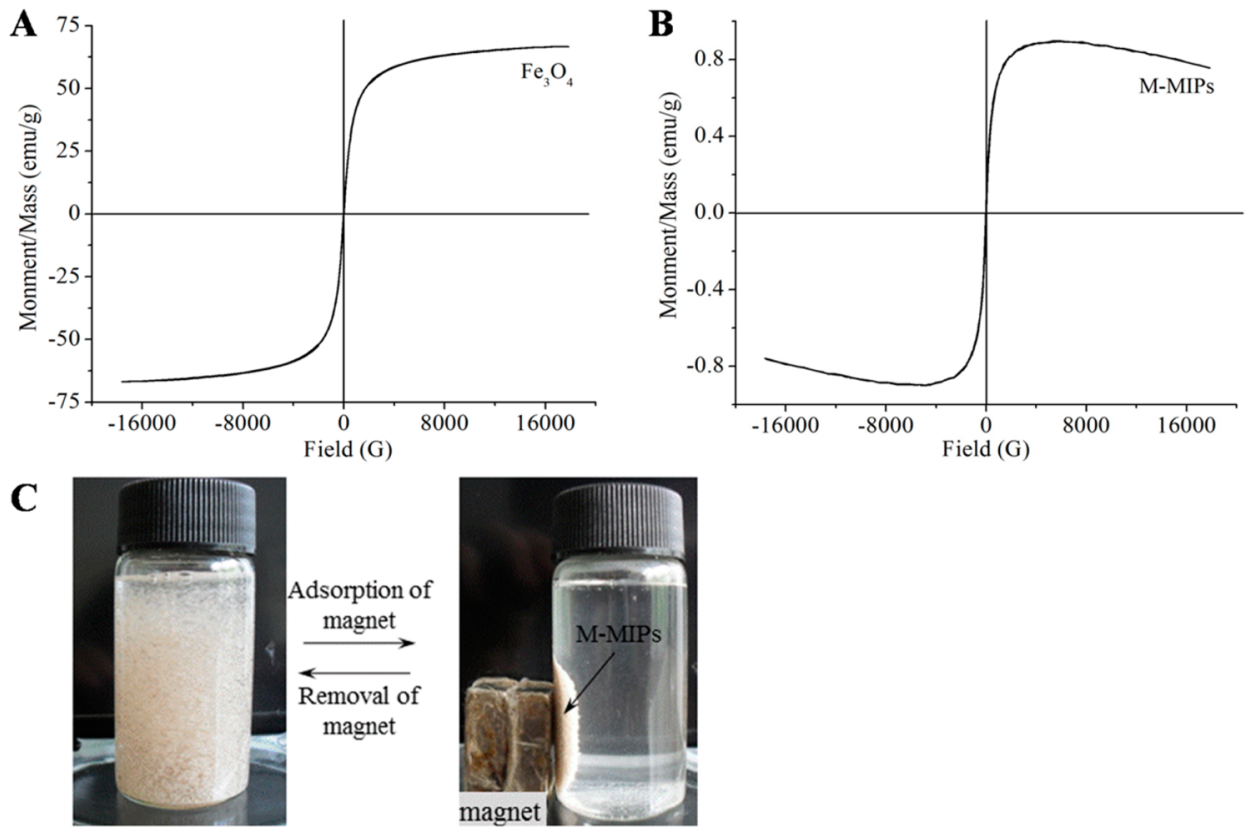

Figure 3. Hysteresis loops of the (A) $\mathrm{Fe}_{3} \mathrm{O}_{4}$ nanoparticle and (B) M-MIPs and (C) magnetic separation picture of M-MIPs.

extracted for $30 \mathrm{~min}$. The solutions were then centrifuged and filtered to obtain the extract solution. The M-MIP particles $(20 \mathrm{mg})$ were added to the extract solution, which was then shaken at room temperature for $1 \mathrm{~h}$. A magnet $\left(\mathrm{N}_{35}\right.$ model $)$ was used to separate the M-MIP particles from the solution. The supernatant was measured by HPLC-UV and determined the amount of vanillin. The food sample without spiking was used as a control group. Thus, the recovery of food samples with spiking has been subtracted by the level of vanillin in the control group. The vanillin adsorbed onto the M-MIP sorbent was subsequently eluted with $2 \mathrm{~mL}$ of methanol/acetic acid $(9: 1, \mathrm{v} / \mathrm{v})$. After desorption for $2 \mathrm{~h}$, the template molecule vanillin and adsorbent were easily and rapidly separated using a magnet. The solution containing the template molecule vanillin was dissolved in $2 \mathrm{~mL}$ of acetonitrile solvent. The recovery amounts of vanillin in food samples were then determined by HPLC-UV. All of the tests were conducted in triplicate, and the data were reported as the mean values. In addition, matrix interferences could be excluded using selective $\mathrm{M}$ MIPs as the extracting agent. The absorbance values were measured by deducting the absorbance of blank samples.

\section{RESULTS AND DISCUSSION}

Preparation of M-MIPs. The water-compatible M-MIPs of vanillin were synthesized via a multi-step polymerization method, as illustrated in Figure 1. Superparamagnetic $\mathrm{Fe}_{3} \mathrm{O}_{4}$ nanoparticles were produced using the co-precipitation method ${ }^{24}$ and then coated with a thin film of silica to enhance the dispersibility of magnetic particles and prevent oxidation. MPS was then allowed to react with the hydroxyl of $\mathrm{Fe}_{3} \mathrm{O}_{4} @$ $\mathrm{SiO}_{2}$ to introduce vinyl groups, which induced the copolymerization of MIPs to occur on the surface of the microspheres upon the addition of MAA. On the other hand, TEOS has a lot of $-\mathrm{OH}$ groups, which can make MPS graft easily onto the $\mathrm{Fe}_{3} \mathrm{O}_{4}-\mathrm{SiO}_{2}$ surface. Finally, the M-MIPs were prepared after the templates were eluted with methanol/acetic acid solution. The obtained M-MIPs enabled the easy recognition, separation, and enrichment of the template from complex food sample samples.

FTIR Spectroscopy. Figure 2A shows the FTIR spectra of $\mathrm{Fe}_{3} \mathrm{O}_{4}, \mathrm{Fe}_{3} \mathrm{O}_{4} @ \mathrm{SiO}_{2}$, and $\mathrm{Fe}_{3} \mathrm{O}_{4} @ \mathrm{SiO}_{2}-\mathrm{MPS}$. The characteristic peak of $\mathrm{Fe}_{3} \mathrm{O}_{4}$ was observed at $580.57 \mathrm{~cm}^{-1}$. The peaks at

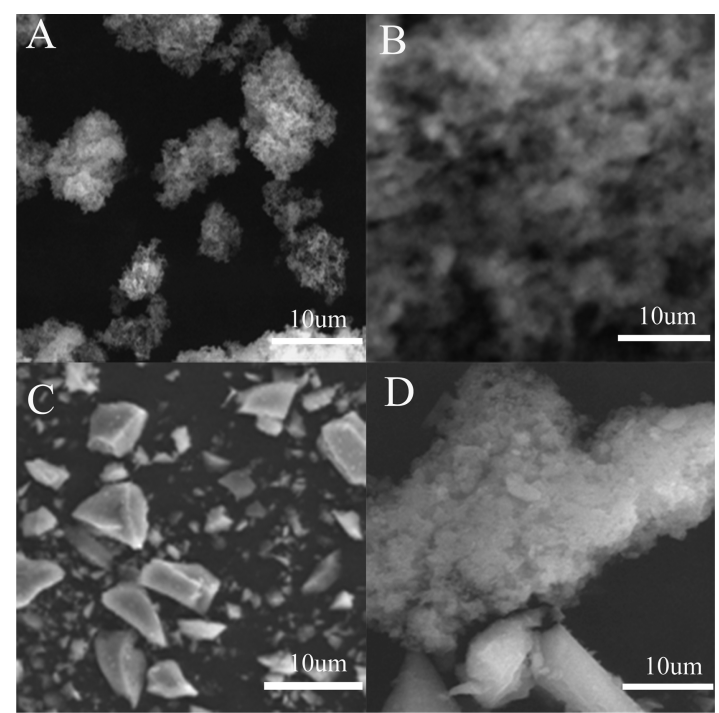

Figure 4. SEM images of (A and B) M-MIPs and (C and D) M-NIPs.

1097.03, 950.00, and $804.40 \mathrm{~cm}^{-1}$ were attributed to the stretching vibrations of $\mathrm{Si}-\mathrm{O}-\mathrm{Si}, \mathrm{Si}-\mathrm{O}-\mathrm{H}$, and $\mathrm{Si}-\mathrm{O}$, respectively, indicating the formation of the silica shell. The absorbance peaks from 2800 to $3000 \mathrm{~cm}^{-1}$ were attributed to the stretching vibrations of the $\mathrm{C}-\mathrm{H}$ bond from the methyl and methylene groups. The peak at $1719.73 \mathrm{~cm}^{-1}$ was attributed to the stretching vibration of the carbonyl group, which indicated the successful modification of MPS.

Crystalline Structure Analysis. Figure 2B shows the XRD patterns of $\mathrm{Fe}_{3} \mathrm{O}_{4}, \mathrm{Fe}_{3} \mathrm{O}_{4} @ \mathrm{SiO}_{2}$, and M-MIPs. A series of characteristic peaks for $\mathrm{Fe}_{3} \mathrm{O}_{4}$ in the $2 \theta$ range of $10-80^{\circ}$ were observed at $2 \theta=30.32^{\circ}, 35.42^{\circ}, 43.39^{\circ}, 53.60^{\circ}, 57.22^{\circ}$, and $62.79^{\circ}$. These peaks were indexed to the (220), (311), (400), (422), (511), and (440) crystalline planes, respectively, which matched well with the data for magnetite [Joint Committee on Powder Diffraction Standards (JCPDS) card 19-629]. However, a few $\gamma-\mathrm{Fe}_{2} \mathrm{O}_{3}$ particles were obtained because of the 

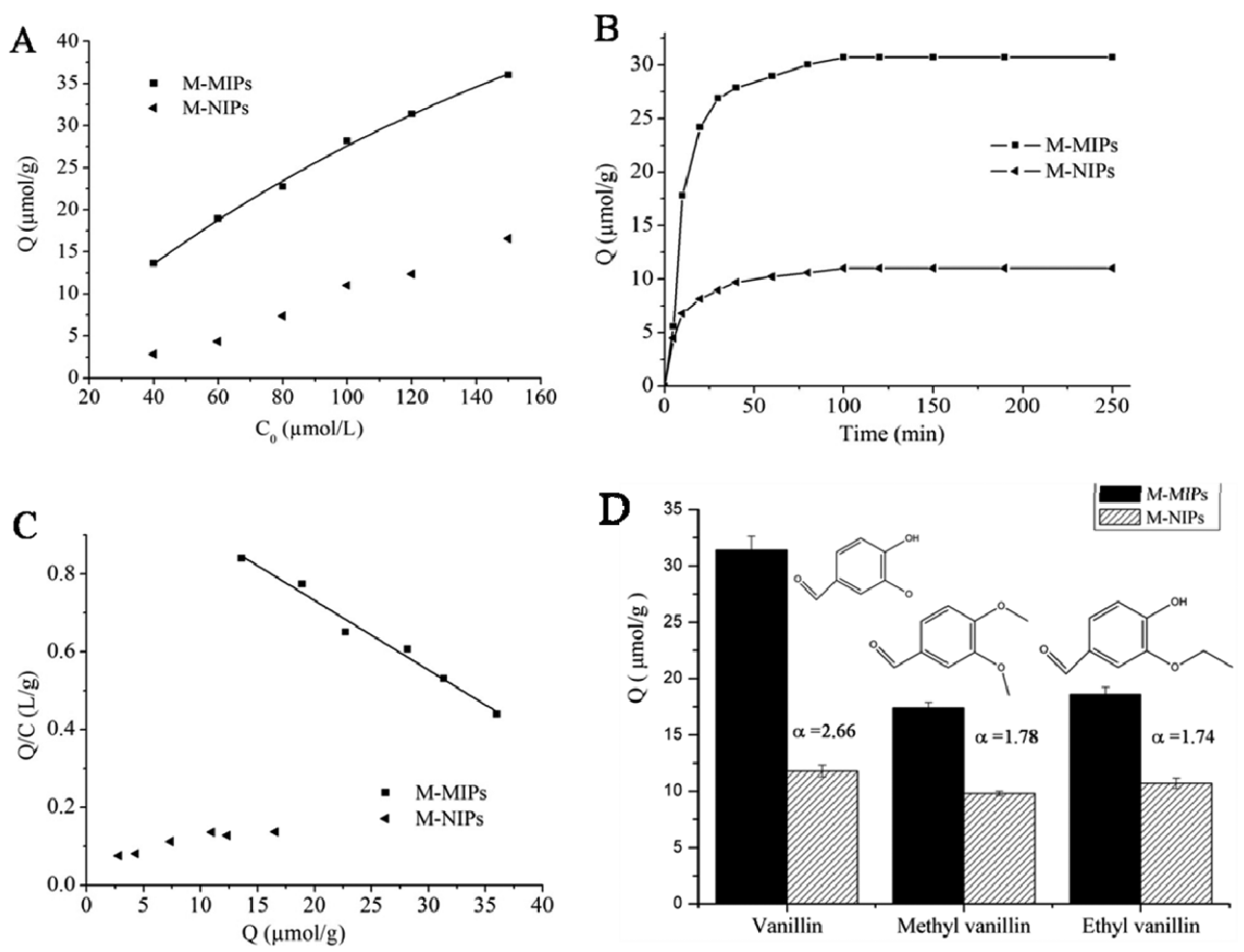

Figure 5. (A) Static adsorption isotherms of M-MIPs and M-NIPs, (B) adsorption kinetic behavior of M-MIPs and M-NIPs, (C) Scatchard analysis plots of M-MIPs and M-NIPs, and (D) competitive binding of vanillin, methyl vanillin, and ethyl vanillin on M-MIPs and M-NIPs.

Table 1. M-MIP Recoveries (\%) and Relative Standard Deviations (RSD \%) Obtained from Analysis of Four Different Samples Spiked with Vanillin

\begin{tabular}{|c|c|c|c|c|c|c|}
\hline \multirow[b]{3}{*}{ sample } & \multicolumn{6}{|c|}{ spiked concentration $\left(\mathrm{mmol} \mathrm{L}^{-1}\right)$} \\
\hline & \multicolumn{2}{|c|}{0.01} & \multicolumn{2}{|c|}{0.04} & \multicolumn{2}{|c|}{0.08} \\
\hline & M-MIPs & M-NIPs & M-MIPs & M-NIPs & M-MIPs & M-NIPs \\
\hline bread & $83.39^{a} \pm 4.27^{b}$ & $42.37 \pm 4.21$ & $92.52 \pm 3.56$ & $38.76 \pm 2.91$ & $91.27 \pm 3.45$ & $36.48 \pm 4.25$ \\
\hline milk powder & $86.23 \pm 5.17$ & $33.41 \pm 3.48$ & $85.35 \pm 3.98$ & $32.84 \pm 4.12$ & $89.97 \pm 4.79$ & $44.96 \pm 3.58$ \\
\hline biscuit & $91.46 \pm 3.49$ & $37.72 \pm 4.33$ & $89.37 \pm 2.72$ & $39.57 \pm 4.38$ & $95.58 \pm 2.48$ & $47.39 \pm 2.94$ \\
\hline chocolate & $89.27 \pm 3.13$ & $41.45 \pm 3.72$ & $92.37 \pm 3.28$ & $32.49 \pm 4.79$ & $92.37 \pm 4.18$ & $47.32 \pm 3.68$ \\
\hline
\end{tabular}

${ }^{a}$ Average value from three individual experiments. The recovery of the food sample with spiking has been subtracted by the level of vanillin in the control group. ${ }^{b} n=3$.

oxidation of $\mathrm{Fe}_{3} \mathrm{O}_{4}$ during the co-precipitation and silanization processes. $^{25-27}$ This finding was not considered in this study, because these particles exhibited similar magnetic properties and either phase allows for the growth of the silica shell on its surface. $^{28}$ Furthermore, by comparing the XRD patterns of $\mathrm{Fe}_{3} \mathrm{O}_{4}$ and $\mathrm{Fe}_{3} \mathrm{O}_{4} @ \mathrm{SiO}_{2}$, some other characteristic peaks for $\mathrm{SiO}_{2}$ were observed in the $2 \theta$ range of $10-35^{\circ}$. Meanwhile, a few peaks could be observed in the M-MIP curve for thick MIPs, which significantly decreased the content of $\mathrm{Fe}_{3} \mathrm{O}_{4} @$ $\mathrm{SiO}_{2}$ in all of the samples. This inference can be validated by the results of thermogravimetric analysis (TGA) and VSM.

TGA. The thermogravimetric curves of $\mathrm{Fe}_{3} \mathrm{O}_{4} @ \mathrm{SiO}_{2}$ and $\mathrm{M}$ MIPs are shown in Figure 2C, which quantified the encapsulation efficiency of the $\mathrm{Fe}_{3} \mathrm{O}_{4} @ \mathrm{SiO}_{2}$ particles that were covered with the M-MIPs. When the temperature increased to $200{ }^{\circ} \mathrm{C}$, the weight of both samples decreased by approximately $4.21 \%$, because of dehydration of the physically adsorbed water or solvent residues. A flat curve of $\mathrm{Fe}_{3} \mathrm{O}_{4} @ \mathrm{SiO}_{2}$ was then observed from 200 to $800{ }^{\circ} \mathrm{C}$. However, the MIPs decomposed rapidly at temperatures of $>800{ }^{\circ} \mathrm{C}$. The decrease in mass was $89.83 \%$, especially from 300 to $500{ }^{\circ} \mathrm{C}$. The residual weight $(5.96 \%)$ was attributed to the thermostable $\mathrm{Fe}_{3} \mathrm{O}_{4} @ \mathrm{SiO}_{2}$.

Magnetic Properties of $\mathrm{Fe}_{3} \mathrm{O}_{4}$ and M-MIPs. The magnetic properties of $\mathrm{Fe}_{3} \mathrm{O}_{4}$ and M-MIPs were determined using VSM at room temperature. The magnetic hysteresis loop of the dried samples is shown in Figure 3. The saturation magnetization $\left(M_{s}\right)$ value of the M-MIPs was significantly decreased to $0.90 \mathrm{emu} \mathrm{g}^{-1}$, in comparison to pure $\mathrm{Fe}_{3} \mathrm{O}_{4}(66.80$ $\left.\mathrm{emu}^{-1}\right)$. The decrease in the magnetization value was due to the formation of thick polymers that covered the M-MIP particles. Moreover, hysteresis did not occur, and remanence and coercivity were zero; thus, both samples were superparamagnetic. As such, the M-MIPs with low $M_{\mathrm{s}}$ values could be easily attracted to an external magnetic field at the wall of the vial (Figure 3C). When the magnet was removed, the $\mathrm{M}$ MIPs were re-dispersed into the solution.

Morphology Analysis. Figure 4 shows the SEM images of the M-MIPs and M-NIPs. In comparison to the irregular and compact structure of the M-NIPs, the structure of the M-MIPs 

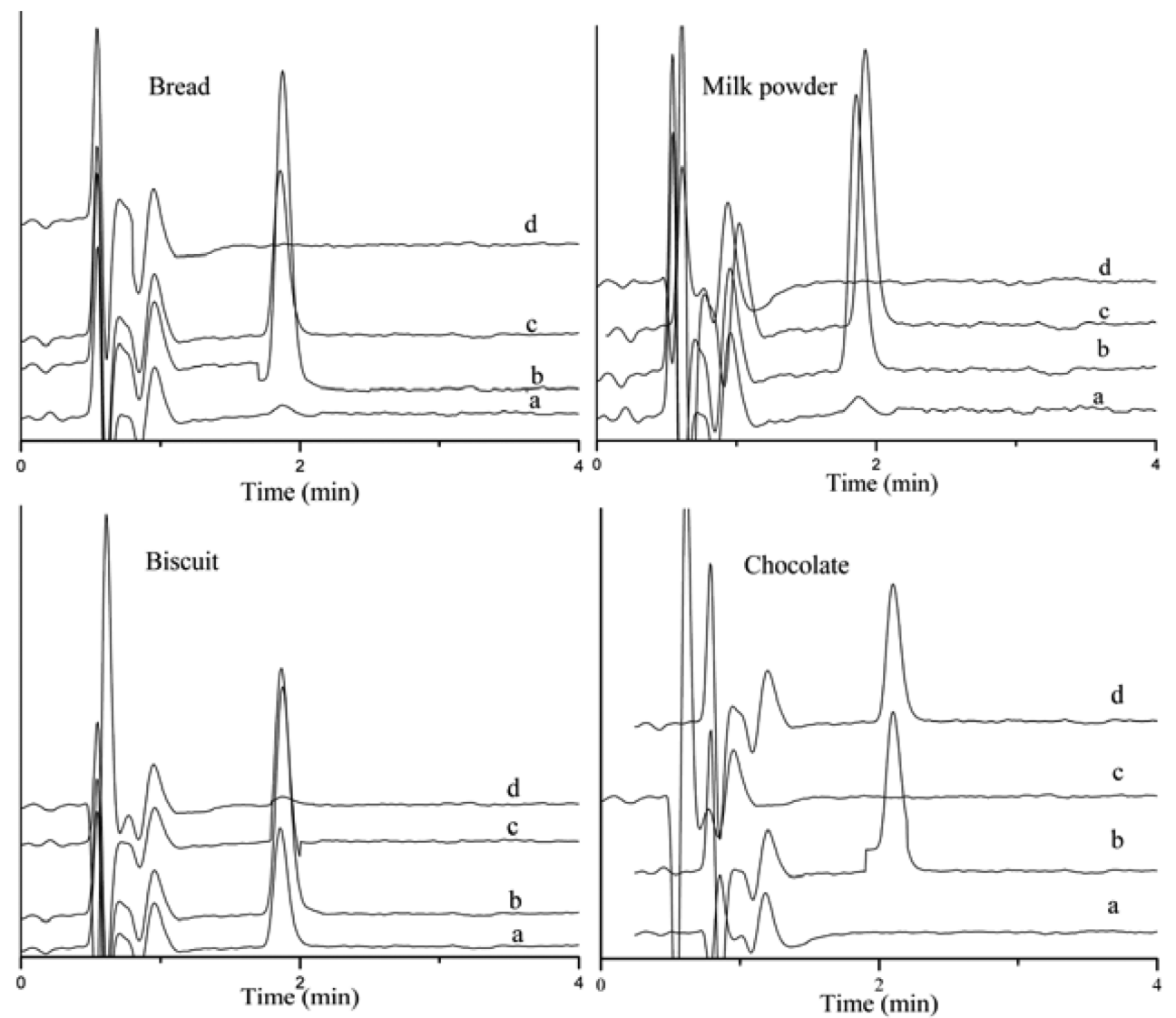

Figure 6. HPLC-UV chromatograms of bread, milk powder, biscuit, and chocolate samples. The samples were (a) not spiked, (b) spiked with 0.01 mmol L ${ }^{-1}$ vanillin and without extraction, (c) spiked with $0.01 \mathrm{mmol} \mathrm{L}^{-1}$ vanillin and with M-MIP extraction, and (d) spiked with $0.01 \mathrm{mmol} \mathrm{L}{ }^{-1}$ vanillin and the remaining solution after extraction.

was loose and porous with many cavities, which facilitated the mass transport and binding kinetics of the template molecules.

Binding Properties of the M-MIPs. To estimate the adsorption capability of the M-MIPs and M-NIPs, static adsorption experiments were conducted using different initial concentrations of vanillin solution. Figure $5 \mathrm{~A}$ shows that the binding amount of the M-MIPs and M-NIPs for vanillin was increased with the increase in the vanillin concentration. Moreover, the amount of vanillin bound to the M-MIPs was much higher than that bound to the M-NIPs. The Scatchard equation was adopted to evaluate the binding ability of the MMIPs and M-NIPs. ${ }^{29}$ The Scatchard equation is expressed as follows:

$$
\frac{Q}{C}=\frac{Q_{\max }}{K_{d}}-\frac{Q}{K_{d}}
$$

where $Q$ is the amount of vanillin bound to M-MIPs at equilibrium, $Q_{\max }$ is the apparent maximum binding amount, $C_{0}$ is the initial concentration of vanillin, $C$ is the concentration of the supernatant at equilibrium, and $K_{\mathrm{d}}$ is the dissociation constant.

As shown in Figure 5C, the Scatchard plot for the M-MIPs was a single straight line with a good linear relationship $\left(R^{2}=\right.$ 0.981), which indicated that the binding sites in the M-MIPs were almost homogeneous. As a control, the M-NIPs with nonlinear correlation possessed fewer homogeneous binding sites. The linear regression equation for the M-MIPs was $Q / C$ $=1.090-0.017 \mathrm{Q}$. The $Q_{\max }$ and $K_{\mathrm{d}}$ were calculated to be 64.12 $\mu \mathrm{mol} \mathrm{g}^{-1}$ and $58.82 \mu \mathrm{mol} \mathrm{L}{ }^{-1}$, respectively, on the basis of the slope and intercept of the Scatchard plot. These values confirmed the high affinity of the M-MIPs to vanillin.

Kinetic adsorption experiments for M-MIPs and M-NIPs were carried out to investigate the binding process. As shown in Figure 5B, the adsorption capacity of M-MIPs was much higher than that of M-NIPs. The adsorption amount of M-MIPs increased rapidly in the first $30 \mathrm{~min}$, and the curve became relatively flat until reaching equilibrium after approximately 100 min. A similar behavior was observed for the M-NIPs in Figure $5 B$. Cavities with recognition sites on the surface of the MMIPs were occupied rapidly by template molecules, which increased the difficulty of transferring vanillin into the inner sites. Given that no specific recognition sites matched vanillin in terms of shape, size, and functional groups in the M-NIPs, the binding amount of the M-NIPs was obviously lower than that of the M-MIPs.

To investigate the binding specificity of the M-MIPs, a selectivity test was conducted using structural analogues of vanillin, methyl vanillin, and ethyl vanillin as control compounds. As shown in Figure 5D, the adsorption capacity of the M-NIPs is similar and non-selective for the three compounds because selective recognition sites are absent in the M-NIPs. However, the M-MIPs showed a significantly higher binding capacity for vanillin than methyl vanillin and ethyl vanillin. The binding capacities of the M-MIPs for vanillin, methyl vanillin, and ethyl vanillin were $31.4,17.4$, and 18.6 $\mu \mathrm{mol} \mathrm{g}{ }^{-1}$, respectively. Accordingly, the imprinting factor values $(\alpha)$ of the M-MIPs for vanillin, methyl vanillin, and ethyl vanillin were $2.66,1.78$, and 1.74 , respectively. These results 
indicated that the M-MIPs exhibited highly specific recognition ability for vanillin. The high selectivity for vanillin may be based on two factors: (1) the first factor is the difference in chemical structures between vanillin, methyl vanillin, and ethyl vanillin, and (2) only vanillin can specifically match the binding sites of the template in terms of size and shape.

Method Validation and Application to the Analysis of Vanillin in Food Samples. To assess the practical applicability of the M-MIPs, four food samples (bread, milk powder, biscuit, and chocolate) were used as real samples in the analysis. At the three spiked vanillin concentrations of 0.01 , 0.04 , and $0.08 \mathrm{mmol} \mathrm{L}^{-1}$, the extraction recoveries of the $\mathrm{M}$ MIPs were $83.39-95.58 \%$ with relative standard deviations of 2.48-5.17\% (Table 1.). The determined limits of detection and quantification were 2.67 and $8.0 \mu \mathrm{mol} \mathrm{L} \mathrm{L}^{-1}$ at signal-to-noise ratios of 3 and 10, respectively. The regression equation was attained, $A_{(279 \mathrm{~nm})}=8.33021 x+2.77378\left(R^{2}=0.9996\right)$ within 0.1-5.0 mmol L $\mathrm{L}^{-1}$. Moreover, the separation process was finished within $10 \mathrm{~s}$ when a magnet was used. The results of HPLC-UV chromatograms (Figure 6) also indicated that MMIPs had high selectivity and enrichment ability.

In summary, novel superparamagnetic particles coated with vanillin-imprinted polymer were synthesized using MAA as the functional monomer and EGDMA as the cross-linker. The resultant M-MIPs showed some attractive characteristics, such as higher binding capacity, faster binding kinetics, and quicker separation. The analytical method based on M-MIPs was successfully applied to vanillin analysis in spiked food samples with higher selectivity and concentration. Thus, the approach offers a simple and straightforward technique for selective separation and fast enrichment of vanillin from complicated food matrices.

\section{AUTHOR INFORMATION}

\section{Corresponding Authors}

*Telephone/Fax: +86-791-86634810. E-mail: peng_hailong@ aliyun.com.

*Telephone/Fax: +86-791-86634810. E-mail: huaxiong100@ 126.com.

\section{Funding}

This work was funded by the National Natural Science Foundation of China (21201098, 51102131, 31160317, 21275158, and 21105117), the Jiangxi Department of Education Fund (GJJ13039), and the Specialized Research Fund for the Doctoral Program of Higher Education (20113601110004).

\section{Notes}

The authors declare no competing financial interest.

\section{REFERENCES}

(1) Zabkova, M.; Otero, M.; Minceva, M.; Zabka, M.; Rodrigues, A. E. Separation of synthetic vanillin at different $\mathrm{pH}$ onto polymeric adsorbent Sephabeads SP206. Chem. Eng. Process. 2006, 45, 598-607.

(2) Walton, N. J.; Narbad, A.; Faulds, C.; Williamson, G. Novel approaches to the biosynthesis of vanillin. Curr. Opin. Biotechnol. 2000, 11, 490-496.

(3) Nadiah, M. Y.; Eri, T.; Takaomi, K. Molecularly imprinted polymer particles having coordinated hydrogen bonding in covalentimprinting for efficient recognition towards vanillin. Sep. Purif. Technol. 2014, 122, 341-349.

(4) Peng, H. L.; Wang, S. Q.; Zhang, Z.; Xiong, X.; Li, J. H.; Chen, L. X.; Li, Y. B. Molecularly imprinted photonic hydrogels as colorimetric sensors for rapid and label-free detection of vanillin. J. Agric. Food Chem. 2012, 60, 1921-1928.

(5) Zhang, G. W.; Ni, Y. N. Simultaneous spectrophotometric determination of vanillin and ethyl maltol in food by multivariate calibration approach. J. Anal. Sci. 2005, 01, 20-23.

(6) Su, L. Q.; Guo, X. L.; Han, S. A. Preparation and evaluation of vanilli molecularly imprinted polymer microspheres by reversible addition-fragmentation chain transfer precipitation polymerization. Anal. Methods 2014, 6, 2512-2517.

(7) Dong, Z. Z.; Gu, F. L.; Xu, F.; Wang, Q. H. Comparison of four kinds of extraction techniques and kinetics of microwave-assisted extraction of vanillin from Vanilla planifolia Andrews. Food Chem. 2014, 143, 54-61.

(8) Waliszewski, K. N.; Pardio, V. T.; Ovando, S. L. A simple and rapid HPLC technique for vanillin determination in alcohol extract. Food Chem. 2007, 101, 1059-1062.

(9) DeJager, L. S.; Perfetti, G. A.; Diachenko, G. W. Comparison of headspace-SPME-GC-MS and LC-MS for the detection and quantification of coumarin, vanillin, and ethyl vanillin in vanilla extract products. Food Chem. 2008, 107, 1701-1709.

(10) Trenholm, R. A.; Vanderford, B. J.; Drewes, J. E.; Snyder, S. A. Determination of household chemicals using gas chromatography and liquid chromatography with tandem mass spectrometry. J. Chromatogr. A 2008, 1190, 253-262.

(11) Sostaric, T.; Boyce, M. C.; Spickett, E. E. Analysis of the volatile components in vanilla extracts and flavorings by solid-phase microextraction and gas chromatography. J. Agric. Food Chem. 2000, $48,5802-5807$.

(12) Shen, Y.; Han, C.; Liu, B.; Lin, Z. F.; Zhou, X. J.; Wang, C. J.; $\mathrm{Zhu}, \mathrm{Z}$. N. Determination of vanillin, ethyl vanillin, and coumarin in infant formula by liquid chromatography-quadrupole linear ion trap mass spectrometry. J. Dairy Sci. 2014, 97, 679-686.

(13) Song, X. L.; Xu, S. F.; Chen, L. X.; Wei, Y. Q.; Xiong, H. Recent advances in molecularly imprinted polymers in food analysis. J. Appl. Polym. Sci. 2014, 131, 40766.

(14) Jiang, T.; Zhao, L.; Chu, B.; Feng, Q.; Yan, W.; Lin, J. M. Molecularly imprinted solid-phase extraction for the selective determination of $17 \beta$-estradiol in fishery samples with high performance liquid chromatography. Talanta 2009, 78, 442-447.

(15) Byun, H. S.; Youn, Y. N.; Yun, Y. H.; Yoon, S. D. Selective separation of aspirin using molecularly imprinted polymers. Sep. Purif. Technol. 2010, 74, 144-153.

(16) Guo, Y.; Wang, R. Y.; Chi, W. H.; Liu, S. A.; Shi, H. G.; Guo, T. Y. One-step synthesis of reactant-product-dual-template imprinted capsules as phosphotriesterase mimetic enzymes for pesticide elimination. RSC Adv. 2014, 4, 7881-7884.

(17) Wang, S.; Peng, H.; Xiong, H.; Ruan, X.; Huang, S.; Dong, L. Preparation and recognition mechanism of vanillin molecularly imprinted polymer microspheres. Food Sci. 2012, 33, 1-7.

(18) Wang, G. S.; Cao, Q. E.; Xiong, J.; Zhu, X. F.; Hou, N. B.; Ding, Z. T. Preparation and recognition properties of vanillin-imprinted polymers. Helv. Chim. Acta 2006, 89, 3032-3040.

(19) Kong, X.; Gao, R.; He, X.; Chen, L.; Zhang, Y. Synthesis and characterization of the core-shell magnetic molecularly imprinted polymers $\left(\mathrm{Fe}_{3} \mathrm{O}_{4} @ \mathrm{MIPs}\right)$ adsorbents for effective extraction and determination of sulfonamides in the poultry feed. J. Chromatogr. A 2012, 1245, 8-16.

(20) Lin, Z.; Cheng, W.; Li, Y.; Liu, Z.; Chen, X.; Huang, C. A novel superparamagnetic surface molecularly imprinted nanoparticle adopting dummy template: An efficient solid-phase extraction adsorbent for bisphenol A. Anal. Chim. Acta 2012, 720, 71-76.

(21) Luo, X.; Zhan, Y.; Huang, Y.; Yang, L.; Tu, X.; Luo, S. Removal of water-soluble acid dyes from water environment using a novel magnetic molecularly imprinted polymer. J. Hazard. Mater. 2011, 187, 274-282.

(22) Madrakian, T.; Afkhami, A.; Mahmood-Kashani, H.; Ahmadi, M. Superparamagnetic surface molecularly imprinted nanoparticles for sensitive solid-phase extraction of tramadol from urine samples. Talanta 2013, 105, 255-261. 
(23) Jing, T.; Du, H.; Dai, Q.; Xia, H.; Niu, J.; Hao, Q.; Mei, S.; Zhou, Y. Magnetic molecularly imprinted nanoparticles for recognition of lysozyme. Biosens. Bioelectron. 2010, 26, 301-306.

(24) Dong, L. L.; Peng, H. L.; Wang, S. Q.; Zhang, Z.; Li, J. H.; Ai, F. R.; Zhao, Q.; Luo, M.; Xiong, H.; Chen, L. X. Thermally and magnetically dual-responsive mesoporous silica nanospheres: Preparation, characterization, and properties for the controlled release of sophoridine. J. Appl. Polym. Sci. 2014, 131, 40477.

(25) Ning, F. J.; Peng, H. L.; Li, J. H.; Chen, L. X.; Xiong, H. Molecularly imprinted polymer on magnetic graphene oxide for fast and selective extraction of $17 \beta$-estradiol. J. Agric. Food Chem. 2014, 62, $7436-7443$.

(26) Liu, B.; Han, M.; Guan, G.; Wang, S.; Liu, R.; Zhang, Z. Highly controllable molecular imprinting at superparamagnetic iron oxide nanoparticles for ultrafast enrichment and separation. J. Phys. Chem. C 2011, 115, 17320-1732.

(27) Li, X. Y.; Huang, X. L.; Liu, D. P.; Wang, X.; Song, S. Y.; Zhou, L.; Zhang, H. J. Synthesis of 3D hierarchical $\mathrm{Fe}_{3} \mathrm{O}_{4} /$ graphene composites with high lithium storage capacity and for controlled drug delivery. J. Phys. Chem. C 2011, 115, 21567-21573.

(28) Wang, X.; Wang, L.; He, X.; Zhang, Y.; Chen, L. A molecularly imprinted polymer-coated nanocomposite of magnetic nanoparticles for estrone recognition. Talanta 2009, 78, 327-332.

(29) Fan, J.; Wei, Y.; Wang, J.; Wu, C.; Shi, H. Study of molecularly imprinted solid-phase extraction of diphenylguanidine and its structural analogs. Anal. Chim. Acta 2009, 639, 42-50. 